\title{
Modelamiento y simulación computacional de la red de consultas médicas de un servicio público de salud chileno
}

\author{
Mario Iván Tarride Fernández, ${ }^{1}$ Óscar C. Vásquez ${ }^{1}$ \\ y Julia González Martinic ${ }^{2}$
}

Forma de citar Tarride Fernández MI, Vásquez OC, González Martinic J. Modelamiento y simulación computacional de la red de consultas médicas de un servicio público de salud chileno. Rev Panam Salud Publica. 2010;27(3):203-10.

RESUMEN Objetivo. Modelizar y simular por computadora la red de atención de consultas médicas de un servicio de salud del sistema público chileno.

Métodos. El estudio se realizó bajo el enfoque de la "dinámica de sistemas". Se recolectó información de los establecimientos del servicio de salud y se construyó un modelo de derivaciones. Se determinaron las especialidades a modelizar de acuerdo con la extensión de sus listas de espera. Se definieron las ecuaciones para el cálculo de las atenciones, las derivaciones y la espera. Se diseñaron las bases de datos y se volcó en ellas la información proveniente del servicio. Se programó y validó el modelo, comparándolo con los datos reales. Se simularon escenarios que permitieran reducir la espera mediante el aumento de las horas médicas, el mejoramiento de la capacidad resolutiva en atención primaria y compleja, o ambos.

Resultados. Se modelizaron 22 establecimientos, 1451 flujos de derivaciones y 12 especialidades médicas además de medicina general. Se procesaron 686869 registros de atenciones, derivaciones y listas de espera. La validación arrojó altos coeficientes de determinación y un error porcentual de 10,43\% respecto de las listas de espera reales.

Conclusiones. La metodología mostró su utilidad para modelizar una red asistencial. Se observó un funcionamiento autorreferencial — según la capacidad instalada-, con posibilidades de intervención para mejorar la resolutividad, tanto en los centros de salud primarios como en los centros de especialidades. Un aumento moderado de la capacidad resolutiva y de las horas médicas permitiría la eliminación total de las listas de espera por consulta médica de especialidad.

Palabras clave Servicios de salud; sector público; gestión en salud; simulación por computador; Chile.

La reforma del sistema de salud chileno surgió como respuesta a un conjunto de problemas bien conocidos, tanto por los especialistas como por la pobla-

\footnotetext{
Universidad de Santiago de Chile, Departamento de Ingeniería Industrial, Santiago, Chile. La correspondencia debe dirigirse a Mario Iván Tarride Fernández, Av. Ecuador 3769, Santiago, Chile. Correo electrónico: mario.tarride@usach.cl

2 Universidad de Chile, Facultad de Medicina, Escuela de Salud Pública, Santiago, Chile.
}

ción en general, que se manifestaban principalmente en la insatisfacción de los ciudadanos con la cobertura, la calidad y los costos de la atención. Para abordarlos, se crearon espacios propicios al desarrollo de iniciativas que permitieran ofrecer nuevas y mejores soluciones. El proceso de reforma se basó en cinco principios fundamentales: el derecho de todo habitante a una adecuada atención de salud; la equidad; la solidaridad entre los grupos favorecidos y los vulnerables; la eficiencia en el uso de los recursos, y la participación social (1).

En este contexto, se formularon los objetivos nacionales de salud y se adoptaron diversas medidas para alcanzarlos, entre ellas, el fortalecimiento de la autoridad sanitaria mediante la definición, en el año 2005, de una nueva estructura organizativa y jurídica; el establecimiento de un plan de salud pública; y un régi- 
men de garantías explícitas en salud, expresado en un conjunto de intervenciones orientadas a garantizar el acceso a una atención de salud oportuna y de calidad (1).

Con respecto a la modificación de la autoridad sanitaria, la redefinición de responsabilidades originó algunos ajustes organizativos, en particular en la estructura del Ministerio de Salud, que pasó a tener dos subsecretarías, una de Salud Pública y otra para Redes Asistenciales. Esta última constituye la máxima autoridad del país en materia de gestión integrada de la red de atención sanitaria, compuesta por 28 servicios responsables de la salud de las personas y del cuidado del ambiente de un área geográfica específica, con cierto grado de autonomía en el manejo de sus respectivos establecimientos. Esto significa que cada servicio debe disponer de la capacidad funcional que le permita gestionar su red de asistencia, constituida por hospitales, servicios de urgencia, centros de diagnóstico y tratamientos (CDT), centros de referencia de salud (CRS), consultorios de especialidades y consultorios generales de atención ambulatoria o centros de salud (CES).

El gestor de red, entre otras actividades, debe conocer el funcionamiento de los establecimientos que la integran y lograr que trabajen en forma coordinada; ocuparse del conjunto y no de lo que sucede dentro de cada uno de ellos; articular acciones con otras redes - servicios de salud- del país, incluidos los prestadores públicos y privados; anticiparse a eventuales dificultades; analizar la demanda de atención médica; programar los recursos necesarios para atender la demanda futura; e identificar y resolver los cuellos de botella. Así, la programación en red habrá de entenderse como "el proceso - liderado por el gestor de red-en el cual, todos y cada uno de los actores involucrados, en un marco de interdependencia, ponen en común sus recursos, tareas y aportes, necesidades y voluntades, para anticipar y predecir en forma sistemática la futura actividad y resolutividad del sistema, con la finalidad de asegurar un mejor estado de salud de la población a cargo, en el marco de los recursos que pone a su disposición el Estado" (2).

La magnitud del desafío que afronta el gestor de red lo lleva a buscar herramientas que faciliten su tarea, sobre todo en lo concerniente a la detección y elimi- nación de los cuellos de botella que se producen en la atención de pacientes ambulatorios. En tal sentido, la modelización y simulación de los flujos de pacientes en la red constituye un aporte importante, ya que permite el análisis de diferentes escenarios sin recurrir a experimentos directos que podrían ocasionar ineficiencias de diversa índole (3).

Por consiguiente, y debido a la complejidad de las redes, se considera que la "dinámica de sistemas" (4) es un referente apropiado para encarar este desafío, tanto conceptual como instrumentalmente, pues se ha mostrado apto para describir sistemas pobres en datos y con alto grado de conexión entre sus componentes (5), capturar las relaciones y efectos no lineales de la realimentación en el sistema (6), y evaluar el impacto que provocarían diversas políticas de gestión, con cierto nivel de validación (7). En la práctica, la dinámica de sistemas ha dado buenos resultados en el análisis de procesos intrahospitalarios tales como, entre otros, la correspondencia entre la oferta y la demanda de servicios (8); la evolución del flujo de pacientes y el efecto de las políticas de gestión (9); el impacto de la introducción de una innovación en el sector de la salud (10); el mejoramiento del servicio por medio de la minimización de los tiempos de espera en un laboratorio de diagnóstico (11); la aplicación de determinadas políticas en los distintos niveles de gestión de un centro hospitalario (12); y el rediseño de la arquitectura de las redes para reducir los tiempos de espera (13). A diferencia de los trabajos recién citados, el presente estudio se orienta hacia la representación de la red de atención de un servicio de salud, para facilitar las tareas de gestión. Sin embargo, dadas las dificultades que plantea el desafío, se decidió trabajar solo sobre uno de los subsistemas: la atención ambulatoria por consultas médicas.

Por eso se ha tomado como objeto principal de estudio la modelización y simulación informática de la red de atención de consultas médicas de un servicio de salud chileno. Se seleccionó para ello el Servicio de Salud Metropolitano Central (SSMC) de Santiago, Chile, entre otros motivos, por ser el único que cuenta con CES bajo su jurisdicción (14).

\section{MATERIALES Y MÉTODOS}

El trabajo se estructuró en las siguientes ocho fases.
Fase I: caracterización del SSMC. Se identificaron los establecimientos que componen la red, y sus interrelaciones, a partir de la información proveniente de fuentes oficiales primarias y secundarias: diagnóstico organizativo del servicio (15), cuenta anual 2007 del Director (16), información de sitios web oficiales (17-19) y entrevistas a directivos y funcionarios del servicio de salud. Se construyó un modelo de flujos de llegada y derivaciones de pacientes. Se seleccionó un conjunto representativo de especialidades médicas aplicando el criterio de mayor impacto en las listas de espera.

Fase II: construcción del modelo matemático. Con el enfoque provisto por la "dinámica de sistemas" $(3,7)$, se construyó el diagrama de influencias para determinar el funcionamiento de la red e identificar las variables críticas que podrían generar cuellos de botella. Se estableció el periodo base de análisis, la unidad de tiempo a observar, así como los parámetros y demás supuestos requeridos, para luego modelizar matemáticamente las atenciones y derivaciones mediante ecuaciones de diferencias.

Fase III: definición de bases de datos. De acuerdo con la modelización descrita, se diseñaron las bases de datos que administran los registros, distinguiendo para cada establecimiento los campos y atributos de la información necesaria. Se consideraron planillas de cálculo en $\mathrm{Mi}$ crosoft Excel, dada su facilidad de manejo.

Fase IV: recolección de los datos. Se obtuvo la información relativa a consultas médicas y derivaciones por especialidad y establecimiento, de las bases de datos del SSMC, para alimentar las respectivas bases de datos definidas en la modelización adoptada.

Fase V: procesamiento de los datos. Se depuraron y estandarizaron los datos obtenidos de las bases del servicio, eliminando los registros defectuosos y homologando las diferentes denominaciones de una misma variable o atributo, en particular para las especialidades médicas, incorporándolas luego a las bases diseñadas. Se determinaron los valores de los parámetros del modelo; se calcularon las probabilidades de derivación de pacientes de un establecimiento a otro y de una especialidad a otra; se cuantificaron las listas de espera por es- 
tablecimiento y especialidad; y se calcularon funciones de distribución para las atenciones y derivaciones por especialidad y establecimiento, según día de la semana y estación del año. Cuando no se encontró significación estadística, se construyeron bandas de datos desde donde obtener valores aleatorios para la simulación. En el caso de los CES, las bandas se obtuvieron sumando a las consultas realizadas los rechazos estimados. Para el CDT y el CRS, las bandas se determinaron restando a las atenciones realizadas las cancelaciones hechas por el médico y sumando las no presentaciones de pacientes a la cita médica.

Fase VI: programación computacional. Frente al conjunto de programas disponibles (Ithink, Versim, Powersim, Stella, Arena, entre otros), se utilizó Arena 11.0 (20), por su buen desempeño en trabajos similares (21-24) y su capacidad para simular eventos discretos, en este caso, la dinámica de atención y derivación de pacientes entre establecimientos. Se estudió la lógica y potencialidad de los módulos de programación del software, asociándolos a entes, variables y ecuaciones, así como la asignación de valores desde las bases de datos diseñadas.

Fase VII: validación del modelo. De acuerdo con los procedimientos habituales, se compararon los datos reales y simulados para el año base que se utilizó. Se estudió la representatividad del modelo al ejecutarlo de dos maneras en los CES: con lista de espera inicial o sin ella. La métrica utilizada consideró el coeficiente de determinación $\mathrm{r}^{2}$, el error medio cuadrático (EMC), y la contribución de sus tres componentes: sesgo $\left(\mathrm{U}^{\mathrm{M}}\right)$, varianza $\left(\mathrm{U}^{\mathrm{S}}\right)$ y covarianza (UC) (21). Se midió el tiempo de simulación del modelo.

Fase VIII: simulación y evaluación de los escenarios. Se definieron y simularon tres escenarios de intervención para disminuir las listas de espera en el CDT y el CRS. El primero consideraba el aumento de las horas médicas, el segundo, el mejoramiento de la resolutividad, y el tercero, una mezcla de los dos anteriores.

\section{RESULTADOS}

Se determinó que la red del SSMC estaba compuesta por diez CES, cinco servicios de atención primaria de urgencia (SAPU), un CDT, un CRS con servicio de urgencia adosado y dos hospitales tipo 1 (de máxima complejidad), el Hospital Clínico San Borja Arriarán (HCSBA) y el Hospital de Urgencia Asistencia Pública (HUAP), cada uno con su respectivo servicio de urgencia. La llegada de pacientes en la red se registra en los CES, los SAPU y los servicios de urgencia del CRS y de los hospitales.

El modelo representativo de la red consideró un total de 1451 flujos, que se ha graficado de manera abreviada en la figura 1. De acuerdo con las estadísticas de listas de espera disponibles, $80 \%$ de ellas correspondían a las siguientes 12 especialidades médicas seleccionadas: oftalmología, otorrinolaringología, neurología de adultos, gastroenterología de adultos, cirugía digestiva alta, dermatología, traumatología de adultos, urología de adultos, endocrinología general, cardiología de adultos, psiquiatría de adultos y afecciones broncopulmonares de adultos.

La forma en que opera el sistema se representó con un diagrama de influencias, según se muestra en la figura 2, donde la relación de causalidad indica que si una variable aumenta, entonces la variable afectada (dependiente) puede aumentar (+) o disminuir (-) su valor. El diagrama muestra ciclos de realimentación negativa y positiva, que expresan la complejidad del equilibrio global de la red. Se observa cómo una reducción de las listas de espera en el nivel inferior de la red por el aumento de las atenciones implica un incremento de ellas en el nivel inmediatamente superior, si en este se mantienen constantes las atenciones habituales. En efecto, la disminución de las listas de espera en los CES solo será posible mediante un aumento de las atenciones; sin embargo, esto a su vez incrementa la cantidad de derivaciones hacia el CDT y el CRS, y por consiguiente también la extensión de las listas de espera; he aquí la principal variable crítica de la red.

Se estableció el día como unidad de tiempo, puesto que el comportamiento de la red es de variación diaria y estacional. Se consideró también que el comportamiento de cada día de la semana era similar para una misma estación del año, tomando como periodo base de estudio el año 2007. Se definieron como parámetros las poblaciones asociadas a cada CES, el promedio de consultas médicas por hora, y la cantidad de horas médicas disponibles por especialidad y por establecimiento.
El resultado de la modelización de los cambios diarios en las atenciones, derivaciones y listas de espera, se expresa mediante las siguientes tres ecuaciones de diferencias:

$$
\begin{aligned}
& L E_{q}^{i p}(d+1)=L E_{q}^{i p}(d)+\sum_{a \in \mathrm{An} E S_{q}} \sum_{b \in E S P_{a}} \\
& C M R_{a}^{b q}(d) * \operatorname{Pr}_{i p}^{b a}(d)-C M D_{q}^{i p}(d+1) \quad \text { Para todo } i, p, q \\
& \sum_{a \in \operatorname{PrES}} \sum_{p \in E S P_{a}} \operatorname{Pr}_{b a}^{i p}(d)=1 \\
& C M R_{q}^{i p}(d+1)=\left\{C M D_{q}^{i p}(d+1) \text { si } \sum_{a \in A_{n} E S_{q}} \sum_{b \in E S P_{a}}\right. \\
& C M R_{a}^{b q}(d) * \operatorname{Pr}_{i p}^{b a}(d)- \\
& C M D_{q}^{i p}(d+1) \geq 0 \quad \text { Para todo } i, p, q \\
& C M R_{q}^{i p}(d+1)=\left\{\sum_{a \in \operatorname{AnES}_{q} b \in E S P_{a}} \sum_{a} R_{a}^{b q}(d) * \operatorname{Pr}_{i p}^{b a}(d)\right. \text { si } \\
& \sum_{a \in \operatorname{AnES}_{q} b \in E S P_{a}} C M R_{a}^{b q}(d) * \operatorname{Pr}_{i p}^{b a}(d)- \\
& C M D_{q}^{i p}(d+1)<0 \quad \text { Paratodo } i, p, q
\end{aligned}
$$

Donde:

$a \in \operatorname{An} E S_{q}$ : Índice que representa a cada uno de los establecimientos desde donde se deriva hacia el establecimiento $q$.

$b \in E S P_{a}:$ Índice que representa a cada una de las especialidades del establecimiento $a$.

$\operatorname{Pr}_{s v}^{i p}(d)$ : Probabilidad de que una consulta médica de la especialidad $i$ realizada en el establecimiento $p$ genere una derivación a la especialidad $v$ del establecimiento $s$, el día $d$.

$C M D_{q}^{i p}(d)$ : Cantidad de consultas médicas disponibles para la especialidad $i$ en el establecimiento $p$, destinadas a derivaciones provenientes del establecimiento $q$, el día $d$.

AnES: $\quad$ Conjunto de establecimientos desde donde se deriva hacia el establecimiento $p$.

$\operatorname{PrES} S_{p}$ Conjunto de establecimientos hacia donde deriva el establecimiento $p$.

$E S P_{p}: \quad$ Conjunto de especialidades del establecimiento $p$.

$C M R_{q}^{i p}(d)$ : Cantidad de consultas médicas realizadas en la especialidad $i$ del establecimiento $p$, destinadas a derivaciones provenientes del establecimiento $q$, el día $d$.

$L E_{q}^{i p}(d): \quad$ Lista de espera de la especialidad $i$ en el establecimiento $p$, para derivaciones provenientes del establecimiento $q$, el día $d$. 
FIGURA 1. Modelo de derivaciones de pacientes por consultas médicas en el Servicio de Salud Metropolitano Central de Santiago, Chile

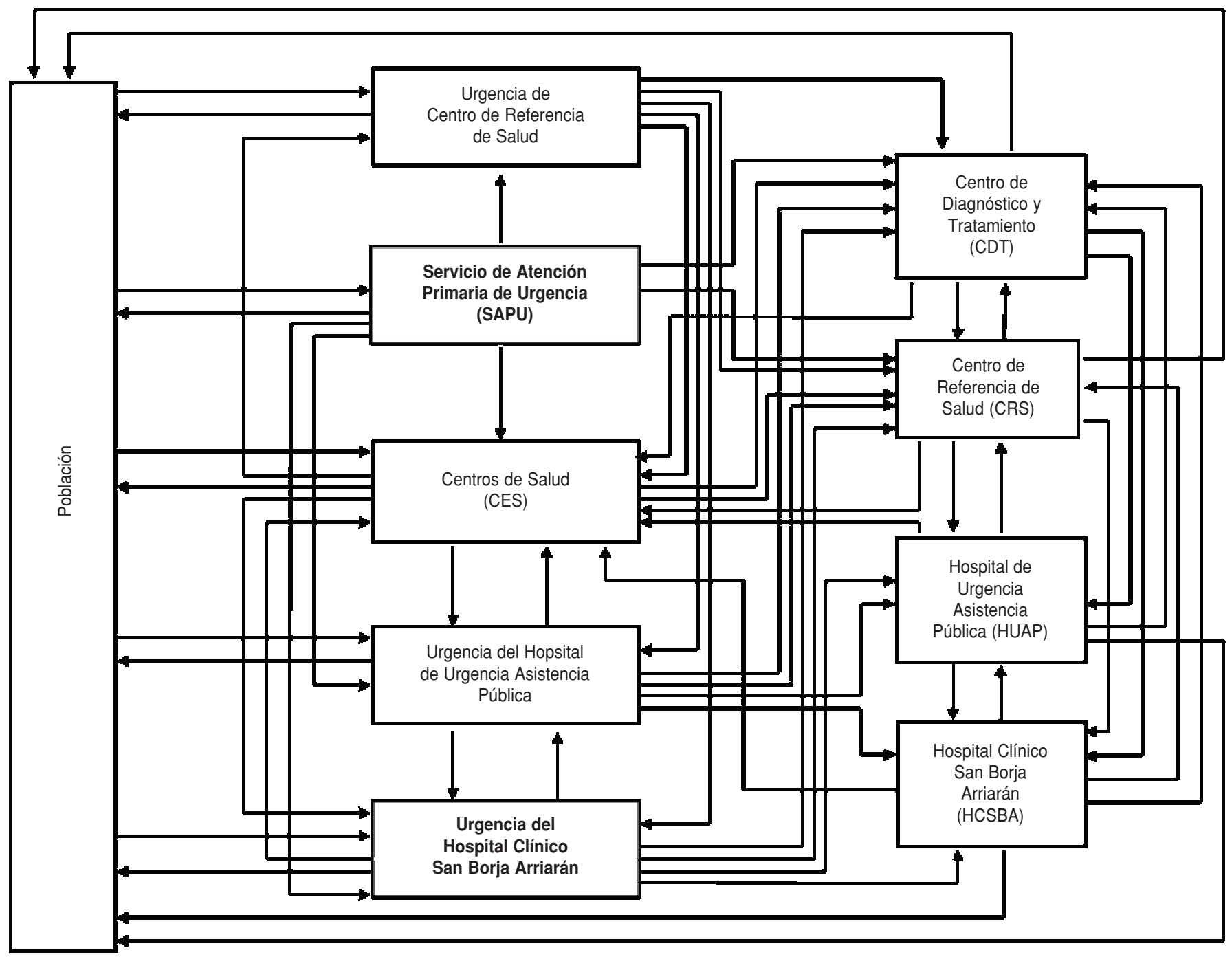

Las bases de datos diseñadas contenían la siguiente información para cada uno de los establecimientos considerados: fecha de la cita médica otorgada al paciente; fecha en que se asignó la cita médica al paciente; especialidad médica de la cita; establecimiento de origen del paciente; estado de la cita, indicando si fue realizada, cancelada o si el paciente no se presentó; establecimiento y especialidad a que fue derivado el paciente.

Las bases de datos para alimentar el modelo quedaron constituidas por 422078 registros de atenciones en CES, 20388 registros con las listas de espera existentes en CES por consultas de especialidad en el CDT y el CRS, 153866 registros con información de las consultas y derivaciones efectuadas en el CDT, y
90537 registros con datos de consultas y derivaciones realizadas por el CRS.

Se determinaron 7448 funciones de distribución para atenciones y derivaciones, de las cuales solo 9,56\% resultaron representativas. En consecuencia, se construyeron las bandas de datos para las consultas realizadas en los CES, que oscilaban entre $10 \%$ y $20 \%$ de amplitud sobre los datos originales. Para el caso del CDT y del CRS las bandas consideraron un porcentaje que oscilaba entre $+7 \%$ $\mathrm{y}-14 \%$ de amplitud respecto de las atenciones originales.

Para la programación en Arena 11.0 se utilizaron los módulos de los paneles del Basic Process, Advanced Transfer y Advances Process. Se asoció la demanda por consulta médica como un Entity Type generado por el módulo Create, la oferta de atención médica de cada establecimiento con el módulo Process; la representación gráfica se realizó con los módulos Station y Route, y la manipulación de registros desde la base de datos en Microsoft Excel con el módulo ReadWrite.

Se validó el modelo simulando el comportamiento del año 2007. Las variables consideradas fueron: consultas realizadas en cada uno de los CES, derivaciones efectuadas en general y por especialidad desde los CES al CDT y al CRS, y consultas brindadas en el CDT y el CRS. Los resultados se presentan en el cuadro 1 .

Para el caso de las consultas realizadas en los CES, sin lista de espera inicial, se obtuvo un $\mathrm{r}^{2}$ de 0,559426 , con un error atribuible principalmente a las covarianzas. En el segundo caso, con lista de espera inicial, el $\mathrm{r}^{2}$ fue de 0,999904 , con un 
FIGURA 2. Diagrama de influencias entre atenciones y derivaciones por consultas médicas en el Servicio de Salud Metropolitano Central de Santiago, Chile

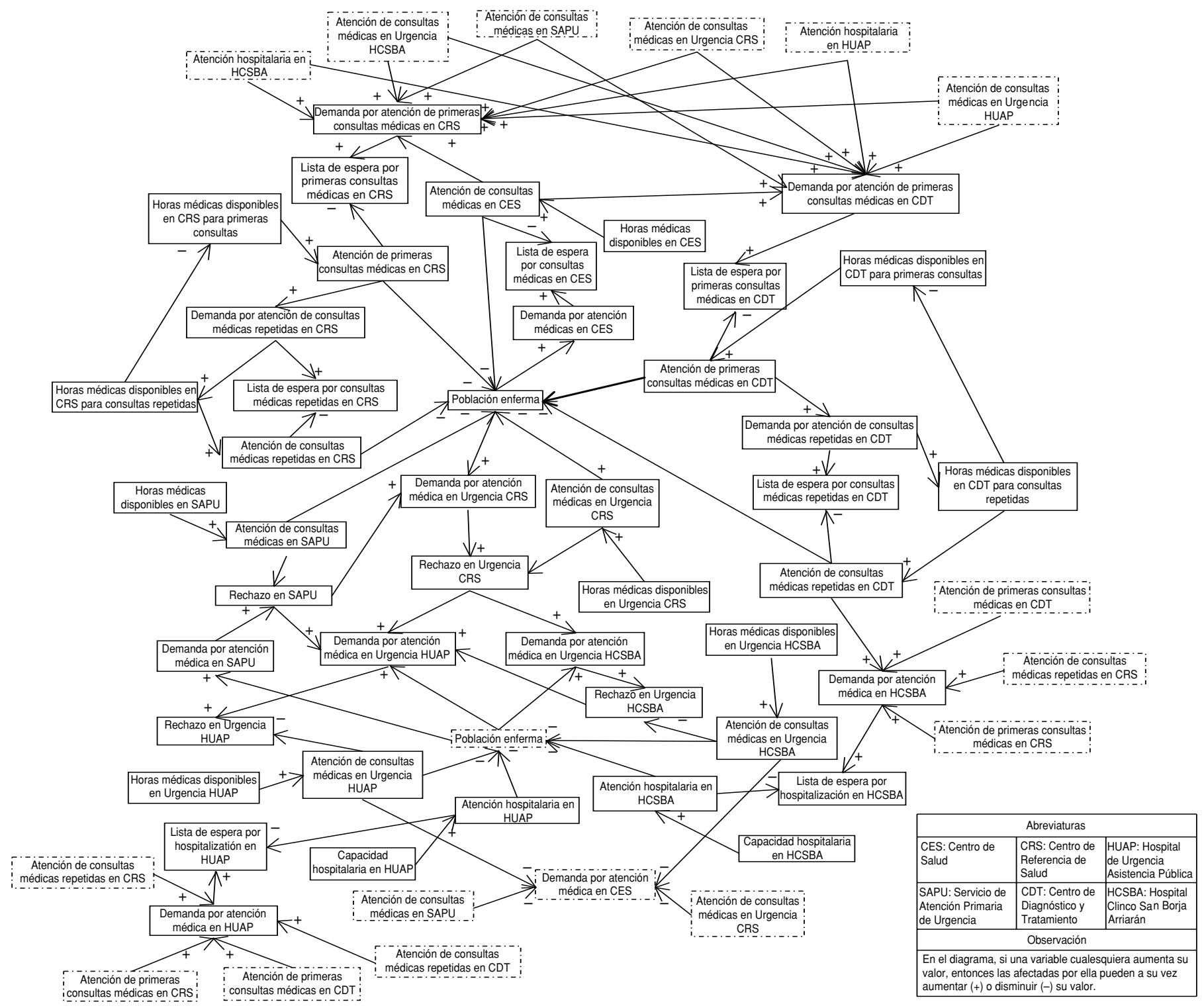

error atribuible casi en su totalidad a la variación en la tendencia central. Considerando que este último caso arrojó mejor coeficiente de correlación respecto de los datos reales, el resto de los resultados se presentan para dicha situación. En relación con el total de consultas derivadas desde cada CES hacia el CDT y el CRS, los valores obtenidos para el $\mathrm{r}^{2}$ fueron 0,88962 y 0,98973 respectivamente, con errores atribuibles, en ambos casos, principalmente a las covarianzas entre las series simuladas y reales. Desde el punto de vista de las especialidades, la agrupación total de las derivaciones desde todos los CES hacia el CDT y el CRS, arrojó un $\mathrm{r}^{2}$ de 0,91263 para el CDT y de 0,76436 para el CRS. En el primer caso, el error es atribuible principalmente a variaciones en la tendencia central, y en el segundo, a las covarianzas entre las dos series. Para el total de consultas realizadas en el CDT y el CRS, los resultados mostraron un $\mathrm{r}^{2}$ de 0,99948 en el caso del CDT, con un error atribuible a las covarianzas, y un $\mathrm{r}^{2}$ igual a 0,59453 con un error atribuible a la diferencia de las tendencias centrales, en el CRS. En promedio, considerando ambos casos, se obtuvo un $\mathrm{r}^{2}$ de 0,78919 , con un error atribuible principalmente a la tendencia central.

La validación del modelo respecto del número de personas en lista de espera al 31 de diciembre de 2007 en el CDT y el CRS, tanto por especialidad como por CES de origen, mostró un error porcentual de 10,43\% entre los datos reales y los simulados.

El tiempo total de simulación del modelo completo resultó de aproximadamente 48 horas (Intel Core 2 Duo, 2,34 GHz, 3,25 GB RAM).

Para el primer escenario, el resultado mostró que el total de horas médicas re- 
CUADRO 1. Coeficientes de determinación y error de validación del modelo de simulación, según consultas y derivaciones realizadas, Chile, 2007

\begin{tabular}{|c|c|c|c|c|c|}
\hline \multirow[b]{2}{*}{ Variable de validación ${ }^{\mathrm{a}}$} & \multirow[b]{2}{*}{$r^{2 b}$} & \multicolumn{4}{|c|}{ Error medio cuadrático ${ }^{\circ}$} \\
\hline & & $E M C$ & $U^{M}$ & $U^{S}$ & $U^{C}$ \\
\hline \multicolumn{6}{|l|}{ Consultas realizadas en CES $^{d}$} \\
\hline Sin lista de espera inicial & 0,559426 & 441525 & 0,20841 & 0,04049 & 0,75109 \\
\hline Con lista de espera inicial & 0,999904 & 19641 & 0,98099 & 0,01800 & 0,00101 \\
\hline \multicolumn{6}{|c|}{ Promedio derivaciones desde CES a: } \\
\hline $\mathrm{CDT}^{\mathrm{e}}$ & 0,88962 & 4268,42 & 0,27606 & 0,00552 & 0,71842 \\
\hline $\mathrm{CRS}^{\dagger}$ & 0,98973 & 4431,92 & 0,21488 & 0,38477 & 0,40036 \\
\hline \multicolumn{6}{|l|}{$\begin{array}{l}\text { Promedio derivaciones por } \\
\text { especialidad desde CES a: }\end{array}$} \\
\hline CDT & 0,91263 & 31,59 & 0,57363 & 0,00766 & 0,41871 \\
\hline CRS & 0,76436 & 2764,93 & 0,11710 & 0,11998 & 0,76292 \\
\hline \multicolumn{6}{|l|}{ Consultas realizadas en: } \\
\hline CDT & 0,99948 & 70,92 & 0,27507 & 0,00010 & 0,72483 \\
\hline CRS & 0,59453 & 135385,75 & 0,58565 & 0,00750 & 0,40684 \\
\hline Total & 0,78919 & 135819,00 & 0,60224 & 0,00782 & 0,38994 \\
\hline
\end{tabular}

a Las tres últimas variables de validación se determinan respecto de la situación inicial con listas de espera, dada su mejor calidad de ajuste.

${ }^{\mathrm{b}}$ El coeficiente de determinación $\left(r^{2}\right)$ muestra el grado de ajuste entre los valores reales y los simulados; varía entre 0 y 1 , magnitud que corresponde al ajuste perfecto.

${ }^{c}$ El error medio cuadrático (EMC) es una medida de la dispersión promedio total entre los valores simulados y los reales. Mientras más cercano a cero, menor es el error. Puede expresarse también como: $E M C=U^{M}+U^{S}+U^{C}$ o bien $E M C=$ $(\bar{S}-\bar{A})^{2}+\left(S_{S}-A_{A}\right)^{2}+2(1-r) S_{S} A_{A}$, donde $\bar{S}$ y $\bar{A}$ son las medias de los datos simulados y reales respectivamente, $S_{S}$ y $A_{A}$ son las desviaciones típicas de cada serie, y r el coeficiente de correlación entre los datos reales y simulados. UM es la fracción de EMC correspondiente a la diferencia entre la media de la serie real y la simulada. US es la fracción de $E M C$ correspondiente a la diferencia entre la desviación típica de la serie real y la simulada. $U^{C}$ es la fracción de EMC correspondiente al ajuste entre la serie real y la simulada, ponderada por sus desviaciones típicas.

d Centro de Salud.

e Centro de Diagnóstico y Tratamiento.

${ }^{\dagger}$ Centro de Referencia de Salud.

queridas para llevar las listas de espera a cero en el CDT y en el CRS era de 5 838. De estas horas, $78,3 \%$ deberían destinarse al CDT y $21,7 \%$ restante al CRS.

Para el segundo escenario, el valor actual promedio de 9,59\% de derivación desde los CES al CDT y al CRS se redujo a $9,15 \%$ y permitió bajar en total $52,61 \%$ las listas de espera en estos dos últimos establecimientos. Tal como se observa en el cuadro 2, la especialidad más afectada fue oftalmología en el CRS, que se redujo en $100 \%$, seguida por traumatología de adultos, con $71,88 \%$. El impacto en el CDT fue de $100 \%$ en cirugía digestiva alta, de 54,77\% en neurología de adultos y de $52,9 \%$ en oftalmología.

El resultado de aumentar en $30 \%$ la resolutividad en consultas repetidas en el CDT y en el CRS significó reducir a cero la casi totalidad de las listas de espera, excepto para las especialidades de oftalmología, otorrinolaringología y neurología de adultos del CDT. No obstante, en promedio, bastaría reducir las consultas repetidas en $17,13 \%$ en el CDT y en $1,62 \%$ en el CRS para obtener resultados similares. La mejora integrada de la resolutividad en los CES, el CDT y el CRS, según los valores antes señalados, permitió llevar casi todas las listas de espera a cero, excepto en oftalmología y neurología de adultos en el CDT.

El tercer escenario, mezcla de los dos anteriores, indicó que un aumento de 2397 horas médicas en el CDT y el CRS permite eliminar sus listas de espera, luego de haber mejorado la resolutividad en los CES. El modelo estimó en 2264 las horas médicas que permiten terminar con las listas de espera que quedaban en oftalmología, otorrinolaringología y neurología de adultos en el CDT, luego de haber disminuido en este las consultas repetidas. Finalmente, aumentando la capacidad resolutiva en los CES y en consultas repetidas del CDT y del CRS, los resultados obtenidos indican que se requieren solo 779 horas médicas

CUADRO 2. Reducción del número de personas en lista de espera por especialidad en el $\mathrm{CDT}^{\mathrm{a}}$ y el $\mathrm{CRS},{ }^{\mathrm{b}}$ según aumento de resolutividad en los CES, ${ }^{\mathrm{C}} 2008$

\begin{tabular}{|c|c|c|c|c|c|c|}
\hline \multirow[b]{2}{*}{ Especialidad } & \multicolumn{2}{|c|}{$\begin{array}{l}\text { Número de personas en } \\
\text { lista de espera inicial }\end{array}$} & \multicolumn{2}{|c|}{$\begin{array}{l}\text { Número de personas en } \\
\text { lista de espera final }\end{array}$} & \multicolumn{2}{|c|}{$\begin{array}{l}\text { \% de reducción de la } \\
\text { lista de espera }\end{array}$} \\
\hline & CDT & CRS & CDT & CRS & CDT & CRS \\
\hline Oftalmología & 1809 & 146 & 852 & 0 & 52,90 & 100,00 \\
\hline Otorrinolaringología & 1433 & 80 & 763 & 37 & 46,76 & 53,75 \\
\hline Neurología de adultos & 1393 & 0 & 630 & 0 & 54,77 & $N A^{d}$ \\
\hline Gastroenterología de adultos & 273 & 0 & 136 & 0 & 50,18 & NA \\
\hline Cirugía digestiva alta & 7 & 346 & 0 & 226 & 100,00 & 34,68 \\
\hline Dermatología & 454 & 524 & 233 & 204 & 48,68 & 61,07 \\
\hline Traumatología de adultos & 158 & 409 & 91 & 115 & 42,41 & 71,88 \\
\hline Urología de adultos & 81 & 108 & 48 & 51 & 40,74 & 52,78 \\
\hline Endocrinología general & 196 & 14 & 98 & 9 & 50,00 & 35,71 \\
\hline Cardiología de adultos & 168 & 51 & 102 & 16 & 39,29 & 68,63 \\
\hline Psiquiatría de adultos & 51 & 0 & 31 & 0 & 39,22 & NA \\
\hline Afecciones broncopulmonares de adultos & 72 & 11 & 41 & 6 & 43,06 & 45,45 \\
\hline Total de establecimientos & 6095 & 1689 & 3025 & 664 & 50,37 & 60,69 \\
\hline Totales generales & \multicolumn{2}{|c|}{7784} & \multicolumn{2}{|c|}{3689} & \multicolumn{2}{|c|}{52,61} \\
\hline
\end{tabular}

a Centro de Diagnóstico y Tratamiento.

b Centro de Referencia de Salud.

c Centro de Salud.

dNA: no se aplica. 
para llevar a cero todas las listas de espera en el CDT y el CRS.

\section{DISCUSIÓN}

El objetivo de modelizar -conceptualmente- y simular - por computadora- la red de atención de consultas médicas del SSMC planteó, entre otros, dos desafíos importantes. El primero, acordar un esquema de derivación de pacientes que conciliara la realidad con la teoría, y las expectativas tanto de los investigadores como de las personas consultadas. El resultado obtenido y expresado en la cantidad de flujos modelizados fue consecuencia del acuerdo entre los participantes, lo que, por cierto, lo hace revisable y modificable. La representación matemática y computacional solo es consecuencia de tal acuerdo. Así, el segundo desafío era, entonces, estimar el grado de validez del modelo programado por computadora, para lo cual se compararon los datos reales de 2007 con los datos simulados para ese mismo año. El primer resultado arrojó un coeficiente de determinación regular, que se explica por haber iniciado la simulación sin listas de espera en los CES y con una tasa de llegada de pacientes menor a la tasa de atención, generándose incluso ocio en los CES. Luego de siete meses de simulación y en virtud del aumento de las tasas de llegada de pacientes y el mantenimiento en su nivel de las tasas de atención, los CES comenzaron a funcionar a plena capacidad, con arreglo a las condiciones reales. Esto mostró la necesidad de iniciar la simulación con tasas de llegada iguales o mayores a las tasas de atención, saturando los CES desde el primer mes de simulación y generando listas de espera. Este esquema de simulación arrojó un coeficiente de determinación excelente; ello indica que la función aleatoria de demanda introducida al modelo por medio de las bandas de datos se comportaba de manera muy parecida a la oferta de atención disponible. Similar calidad de ajuste se observó para las listas de espera en el CDT y el CRS. Puede afirmarse, entonces, la validez tanto de la conceptualización como de la formalización matemática y la programación computacional de la red de consultas médicas del SSMC.

Los errores estadísticos encontrados son atribuibles principalmente a las diferencias entre las medias de las series reales y simuladas, y al valor del ajuste pon- derado por las desviaciones típicas de ambas series. Puede afirmarse entonces que si bien las desviaciones típicas de ambas series — real y simulada- son semejantes, sus curvas no llegan a superponerse y los datos muestran dispersión respecto de su media. Otro factor de error en el modelo puede asociarse con la homologación de denominaciones que fue necesario realizar para algunas especialidades, debido a que las bases de datos utilizaban nombres diferentes.

En un sistema con listas de espera como el estudiado, en el que domina la oferta, no necesariamente se captura el patrón de comportamiento de la demanda. Esto pone de relieve la necesidad de realizar investigaciones que permitan estimar la demanda real que enfrenta la red de atención, no solo para lograr una mejor representatividad del modelo sino también para perfeccionar la planeación y programación de actividades en la realidad.

Por otra parte, el tiempo de simulación relativamente prolongado puede explicarse por la discreta eficiencia del programa informático utilizado. Así, para cada consulta médica a representar, el programa genera una "entidad" que permitiría incluso individualizar al paciente de que se trata. Este hecho, que le otorga al modelo gran especificidad, le resta eficiencia en la simulación de escenarios, al incrementar el tiempo de cómputo.

En cuanto a los escenarios analizados, se aprecia la importancia de intervenir no solo en el nivel donde se encuentra la variable crítica de la red, las listas de espera en el CDT y en el CRS, sino también en otros niveles. En efecto, un aumento de menos de $0,5 \%$ en la capacidad resolutiva en atención primaria tuvo un impacto de reducción en las listas de espera en el CDT y el CRS de más de 50\%.

La mejora de la resolutividad en un $30 \%$ en consultas repetidas en el CDT y el CRS, se obtuvo considerando la posibilidad de reducir de seis a cuatro el número promedio de consultas requeridas para resolver un episodio de enfermedad. Este supuesto debe observarse con cautela, en tanto representa un valor promedio que podría no ser factible para algunas especialidades y diagnósticos.

La investigación realizada presenta la limitación de haber modelizado solamente consultas médicas, dejando afuera las hospitalizaciones, procedimientos $\mathrm{y}$ exámenes de apoyo diagnóstico, así como a los prestadores privados. Sin embargo, el subsistema estudiado mantiene organicidad, aun cuando se haya perdido completitud al no modelizar la red completa. Otra limitación fue no haber podido cerrar algunos ciclos de derivación por no disponer de información para las contrarreferencias, cruciales para estudiar y buscar formas de alcanzar el equilibrio en la red.

\section{Conclusiones}

La experiencia realizada mostró que efectivamente es posible modelizar y simular por computadora la red de atenciones y derivaciones de consultas médicas dentro de un servicio de salud. Los coeficientes de determinación obtenidos mostraron la validez del modelo. El comportamiento de la red modelizada está dominado por la oferta de consultas médicas en atención primaria, desconociéndose la demanda real que enfrenta.

El problema de las listas de espera en atención ambulatoria compleja se explica en un $80 \%$ por la habilitación de solo 12 especialidades médicas.

Mejorar la resolutividad en los CES, el CDT y el CRS de manera conjunta, más un aumento moderado de las horas médicas, demostró ser una solución efectiva al problema de las listas de espera en consultas de especialidad. En todo caso, el mayor impacto se obtuvo al mejorar la capacidad resolutiva en los CES.

Con el objeto de reducir el tiempo total de procesamiento observado, deberían experimentarse otras formas de programación y de software, que permitan suministrar al gestor de redes una herramienta auxiliar eficiente.

Por último, y con el fin de contribuir con investigaciones similares a esta, se recomienda estudiar y conocer la demanda real dirigida a los centros de salud primarios, registrar y mantener datos de contrarreferencias, homologar e integrar la información disponible relativa a atenciones y derivaciones, y ampliar el modelo incorporando hospitalizaciones y procedimientos de apoyo diagnóstico y tratamiento.

Agradecimientos. Este trabajo se realizó bajo el auspicio del Fondo Nacional de Investigación y Desarrollo en Salud (FONIS) /Comisión Nacional de Investigación Científica y Tecnológica (CONICYT) de Chile (Proyecto SA06I20060), y contó con el valioso apoyo del personal del Servicio de Salud Metropolitano Central, Santiago, Chile. 


\section{REFERENCIAS}

1. Chile, Ministerio de Salud. Reforma de la Salud en Chile. Santiago: MINSAL; 2004.

2. Chile, Ministerio de Salud. Orientaciones para la programación en red. Santiago: MINSAL; 2006.

3. Jun JB, Jacobson SH, Swisher JR. Application of discrete-event simulation in health care clinics: a survey. J Oper Res Soc. 1999;50(2):109-23.

4. Forrester JW. Industrial dynamics. Boston: MIT Press and John Wiley and Sons Inc. 1961.

5. Hirsch G. System dynamics modeling in health care. ACM SIGSIM Sim Dig. 1979;10(4): 38-42.

6. Sterman J. Business dynamics: systems thinking and modeling for a complex world. Boston: McGraw-Hill; 2000.

7. Young, T. An agenda for healthcare and information simulation. Health Care Manag Sci. 2005;8(3):189-96.

8. Lubyansky A. A system dynamics model of health care surge capacity. En: Sterman J, Repenning N, Langer R, Rowe JI, Yanni J, eds. Proceeding of the 23rd International Conference of the System Dynamics Society; $2005 \mathrm{Jul}$ 17-21; Boston, USA. Boston: The System Dynamics Society; 2005. Disponible en: http:// www.systemdynamics.org/conferences/ 2005/proceed/papers/LUBYA208.pdf. Acceso el 15 de marzo de 2008.

9. Homer J, Hirsch G. System dynamics modeling for public health: Background and opportunities. Am J Public Health. 2006;96(3): 452-58.

10. Horsh A, Khoshsima D. Towards modeling and simulation of integrated social and health care services for elderly. Medinfo. 2007;12(1): $38-42$.

11. Rohleder T, Baskin L, Bischak, D. 2007. Modeling patient service centers with simulation and system dynamics. Health Care Manag Sci. 2007;10(1):1-12.
12. Wolstenholme E, Monk D, McKelvie D, Arnold S. Coping but not coping in health and social care: masking the reality of running organisations beyond safe design capacity. Syst Dynam Rev. 2007;23(4):371-89.

13. Ying $S$, Zhanming, J. Modeling health service centers with simulation and system dynamics. En: Kawada S, ed. UKSim 2008. Proceedings of the 10th International Conference on Computer Modelling and Simulation; 2008 Apr 1-3; Cambrige, UK. Washington: IEEE Computer Society; 2008. Pp. 649-54.

14. Chile, Ministerio de Salud. Diagnóstico de Salud. Servicio de Salud Metropolitano Central. Santiago: MINSAL; 2004.

15. Chile, Servicio de Salud Metropolitano Central. Cuenta Pública. Santiago: SSMC; 2007.

16. Chile, Centro de Referencia de Maipú. Quiénes somos en el CRS Maipú. Disponible en: http://www.crsm.cl/nosotros.html. Acceso el 5 abril de 2008.

17. Chile, Ministerio de Salud. Conózcanos. Subsecretaría de Redes Asistenciales. Disponible en: http://www.redsalud.gov.cl/portal/url/ page/minsalcl/g_conozcanos/g_subs_redes asist/presentacion_subs_redes_asist.html. Acceso el 9 de abril de 2008

18. Chile, Servicio de Salud Metropolitano Central. Establecimientos. Disponible en: http:// www.ssmc.cl/establecimientos.htm. Acceso el 10 de abril de 2008.

19. Rockwell Automation-Arena Simulation Software. Arena Product Overview. Disponible en: http://www.arenasimulation.com/ products/default.asp. Acceso el 5 de marzo de 2008.

20. Alexopoulos C, Goldsman D, Fontanesi J, Sawyer M, De Guire M, Kopald D, et al. En: BA Peters, JS Smith, DJ Medeiros, MW Rohrer, eds. A discrete-event simulation application for clinics serving the poor. WSC'01.
Proceedings of the 2001 Winter Simulation Conference; 2001 Dec 9-12; Arlington, USA. Baltimore: INFORMS Simulation Society; 2001. Pp. 1386-91.

21. Centeno M, Lee M, Lopez E, Fernandez H, Carrillo M, Ogazo T. A simulation study of the labor and delivery rooms at JMH. En: BA Peters, JS Smith, DJ Medeiros, MW Rohrer, eds. WSC'01. Proceedings of the 2001 Winter Simulation Conference; 2001 Dec 9-12; Arlington, USA. Baltimore: INFORMS Simulation Society; 2001. Pp. 1392-400.

22. Ramis F, Palma J, Baesler F. The use of simulation for process improvement at an ambulatory surgery center. En: BA Peters, JS Smith, DJ Medeiros, MW Rohrer, eds. WSC'01. Proceedings of the 2001 Winter Simulation Conference; 2001 Dec 9-12; Arlington, USA. Baltimore: INFORMS Simulation Society; 2001. Pp. 1401-4.

23. Ramakrishnan $S$, Nagarkar $K$, DeGennaro $M$, Srihari K, Courtney A, Emick F. A study of the $\mathrm{CT}$ scan area of a healthcare provider. En: RG Ingalls, MD Rossetti, JS Smith, and BA Peters, eds. WSC'04. Proceedings of the 2004 Winter Simulation Conference; 2004 Dec 5-8; Washington D.C., USA. Baltimore: INFORMS Simulation Society; 2004. Pp. 2025-31.

24. Oliva R. A VENSIM module to calculate summary statistics for historical fit. Boston: Sloan School of Management-MIT; 1997. Report N D-4584.

Manuscrito recibido el 7 de mayo de 2009. Aceptado para publicación, tras revisión, el 9 de septiembre de 2009.
ABSTRACT

Computer modeling and simulation of the patient-visit network within a Chilean public health service

Key words
Objective. To create a computerized model and simulation of the patient visit/ referrals process for a health care services organization within Chile's public health care system.

Methods. The study was performed with a "system dynamics" focus. Data were collected from the health care service centers, and a referrals model was created. Specialty areas to be modeled were chosen based on the length of their waiting times. Equations were defined for calculating care visits, referrals, and waiting times. Databases were designed and populated with centers' data. The model was programmed and validated through comparisons with actual data. Scenarios were simulated that would reduce patient waiting time by increasing staff hours or improving diagnostic resolution at the primary care and specialty centers, or both.

Results. The modeling included 22 centers, 1451 referral flows, and 12 medical specialty areas, in addition to general medicine. A total of 686869 registered office visits, referrals, and waiting logs were processed. The validation yielded high determinant coefficients and a $10.43 \%$ percentage error regarding actual waiting time logs.

Conclusions. This methodology proved to be useful for modeling a health care network. An auto-referral process was observed where options were available, with opportunities for improvement in diagnostic resolution at the primary health care centers, as well as at the specialty centers. Moderate increases in diagnostic accuracy and office hours would completely eliminate waiting times at the specialty centers.
Health services; public sector; health management; computer simulation; Chile. 